\title{
Kaizen and ergonomics: the perfect marriage
}

\author{
Martin Antonio Rodriguez ${ }^{\mathrm{a},{ }^{*}}$, Luis Fernando Lopez ${ }^{\mathrm{b}}$ \\ ${ }^{a}$ Safety and Health, Toyota Argentina S.A., Ruta $12 \mathrm{Km}$ 81, CP 2800, Zarate, Pcia. Buenos Aires, Argentina. \\ ${ }^{\mathrm{b}}$ Human Resources, Toyota Argentina S.A., Ruta $12 \mathrm{Km}$ 81, CP 2800, Zarate, Pcia. Buenos Aires, Argentina.
}

\begin{abstract}
This paper is an approach of how Kaizen (Continuous Improvement) and Ergonomics could be implemented in the field of work. The Toyota's Team Members are the owners of this job, applying tools and techniques to improve work conditions using the Kaizen Philosophy in a QCC Activity (Quality Control Circle).
\end{abstract}

Keywords: Kaizen, Ergonomics, Toyota, Argentina.

\section{Introduction}

Kaizen philosophy (continuous improvement) is the base of any kind of work in Japanese companies. All the industrial workers (also known as Team Members) are committed to Kaizen, participating in activities such as suggestions or QCC: Quality Control Circles. A QCC consist in a Work Team which solves complex problems or improve conditions with specific techniques while Suggestions are Ideas that solve problems or improve conditions in an easy and quick way. In Fig. 1 this relationship is showed.

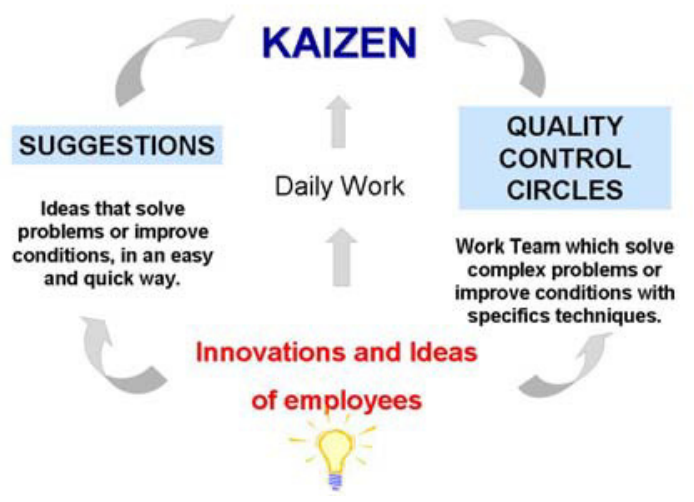

Fig. 1: Kaizen, Suggestions and Quality Control Circles.
But there is an interesting point about QCC activities; every year more than 500 people are involved in Ergonomics QCC. Ergonomics and Kaizen combined in one company strategy philosophy are the key tool to develop company's safety culture plan.

The two keys of Toyota way are:

- Continuous improvement

- Respect

QCC activities are an effective way to put into practice the Toyota Way, as shown in Fig. 2

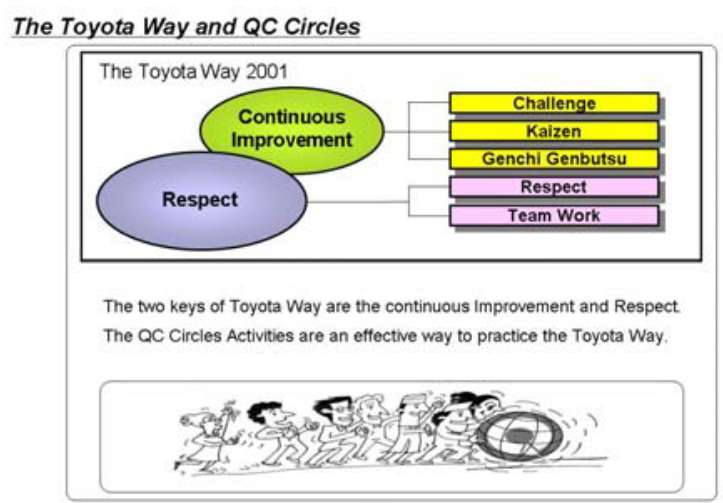

Fig. 2: The Toyota Way and QC Circles.

\footnotetext{
*Corresponding author. E-mail: martinrodri@yahoo.com
} 


\section{Project Organization}

The activity consists in a group of pre-established meetings in which Team Members discuss, create, plan and solve all kinds of problems related to ergonomics. The important thing here is that they work on their own, with a QCC Team Leader and with a QCC Theme Leader.

The main purposes of QCC is:

- To improve personal performance

- To promote a better work environment

- To support the company development

In the beginning, each Team Member of the Kaizen Circle Group is assessed on issues such as Working environment, team work, problem solving tools, quality conscience, quality tools, etc., as shown as in Fig. 3. Then they evaluate different kinds of problems in their workplace to find the main important issue. Ergonomics is one of the most important issue that team members select.

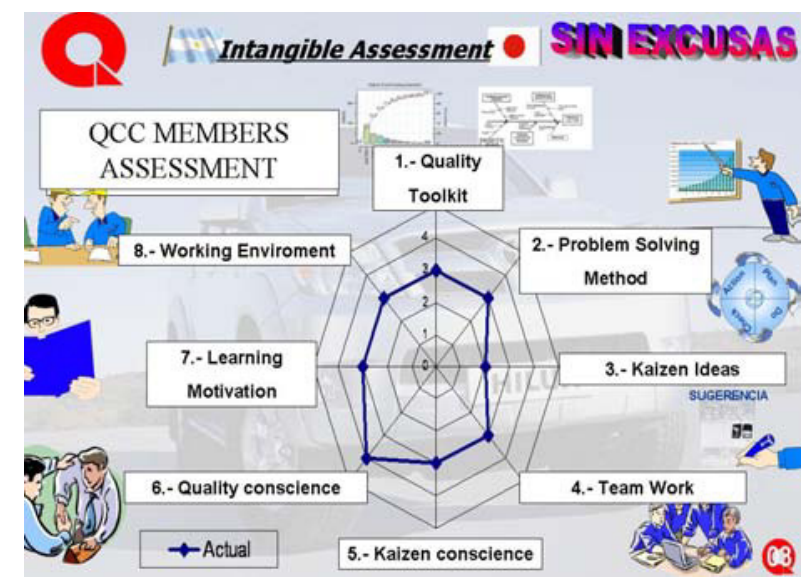

Fig. 3: Initial Intangible Assessment

Then, team members define a QCC target, which in general, it consists in the elimination of ergonomics problems such as bad postures, high efforts or repetitive movements. This is shown in Fig 4.

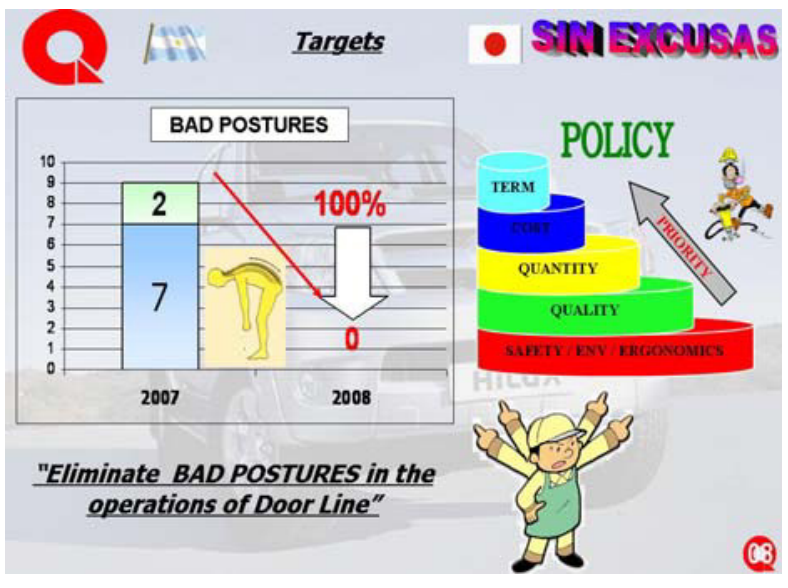

Fig. 4: Activity Target: Bad postures reduction.

Company's Ergonomist helps groups to evaluate jobs and operations, giving advice and general solutions to the possible cause, developing countermeasures by themselves.

\section{Human Factors topics covered}

They use the Ishikawa diagram to root out the main causes that produce the problem. Main causes in general are connected to lack of training, bad postures, workplace design problems, tools, methods, etc. In Fig. 5 there is an example of that kind of analysis. They also apply the 5 Why Technique to find the root cause, consisting on making the famous question five times: Why? Why? Why? Why? Why?

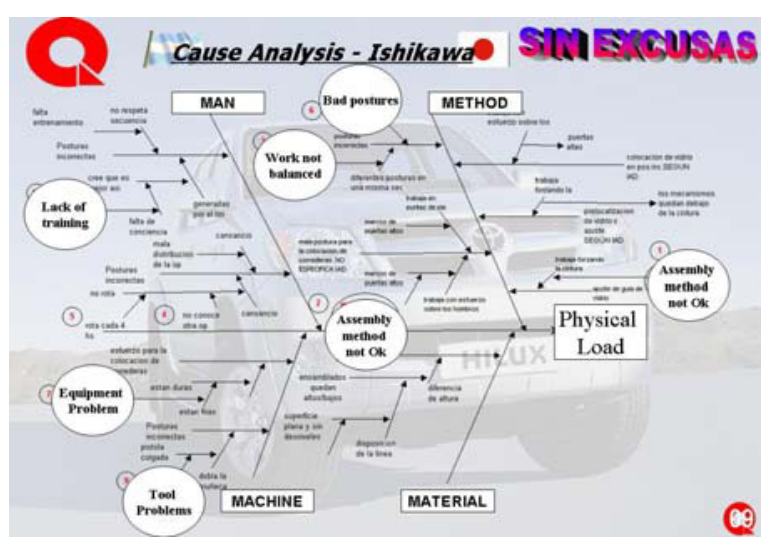

Fig. 5: Root Cause Analysis - Ishikawa Diagram 
Then, they prepare a list of causes that produce the main problem, for example: Bad postures, and they establish a connection between the cause and the countermeasure that must be developed to reduce the source impact. Please refer to Fig. 6 to view a list of causes and possible solutions.

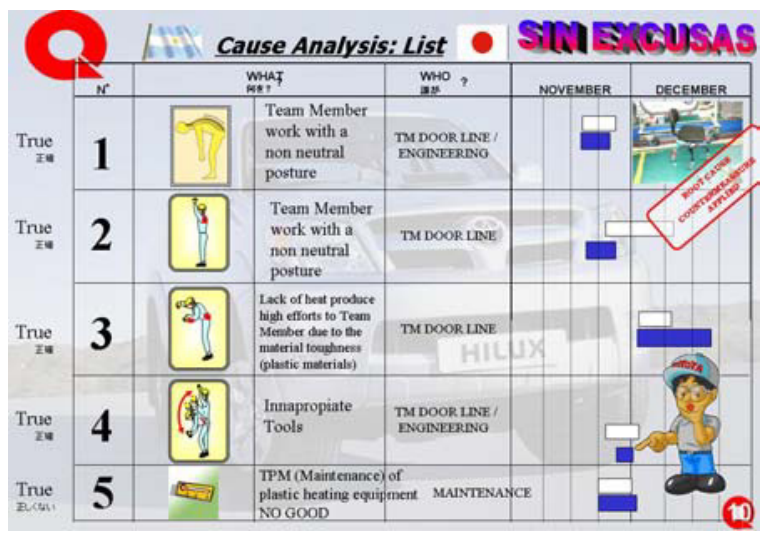
dule

Fig. 6: List of Causes and problem solving sche-

\section{Project Phases}

When the team members have their solutions in mind, they have to prepare schedule with all the actions that must be taken. All this actions are studied together with process engineers, who help team members to implement countermeasures that entail investments or big changes. Refer to Fig. 7.

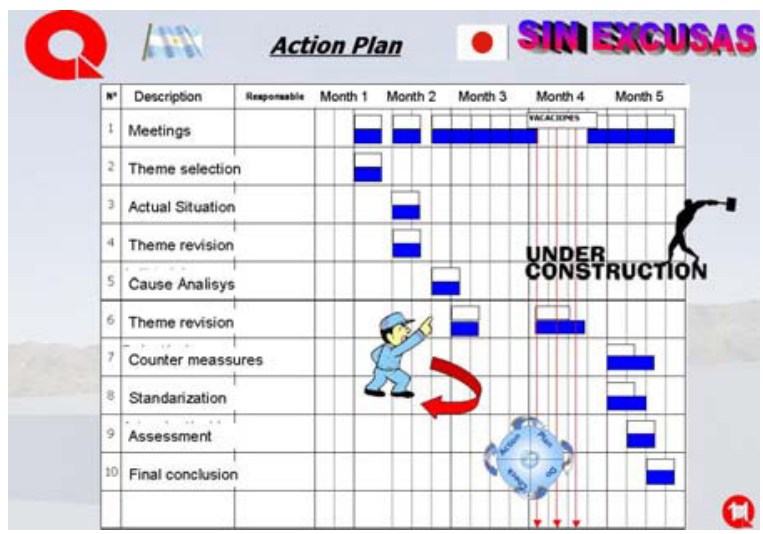

Fig. 7: Action Plan of countermeasures.

In the following figure the Team Members use the Before and After pictures taken in the workplace, after taking the countermeasure. Please note that the body main posture was improved due to a pit installation in the workplace and the introduction of platforms(see Fig. 8).

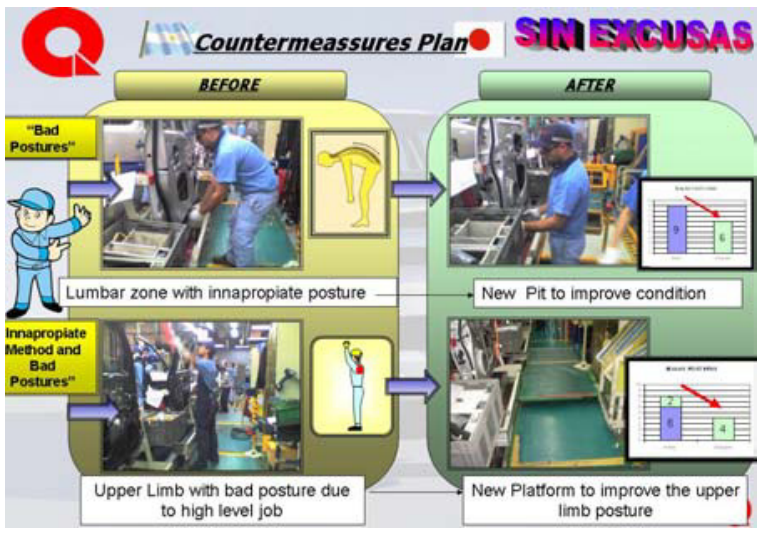

Fig. 8: Images of countermeasures taken in the workplace.

Finally, the team members assessed the initial intangible assessment variables again. In Fig. 9 you may see that an important improvement was achieved, where the red line shows the previous condition and the blue line shows the later condition.

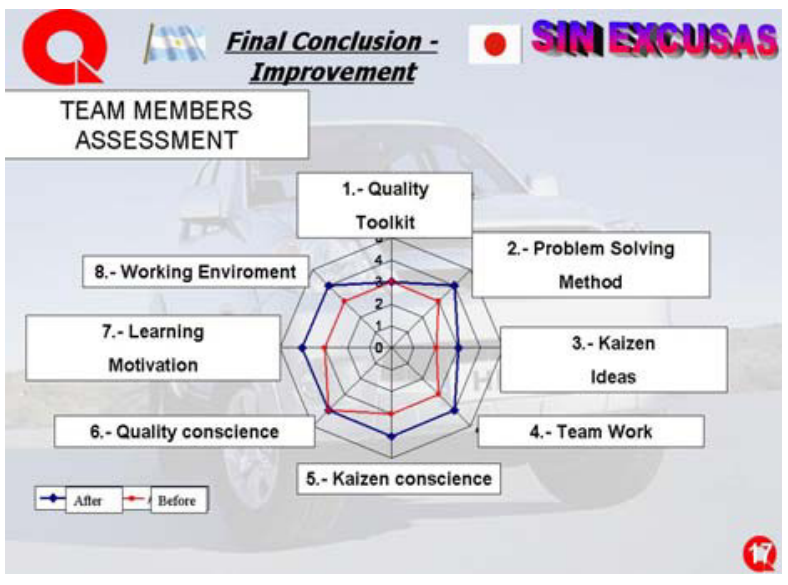

Fig. 9: Final Intangible Assessment

\section{Conclusion}

This work is done by trained Team Members. They solve problems and improve process by their own. Engineers and Ergonomists supports them when 
they need a method or a special advice, but the leadership and the attitude of TM's Job is admirable.

Ergonomist and Engineers only train them on how to fish, and this is the fishing. 